\title{
Etude des variations des teneurs de quelques oxydes de deux types de sols à plinthites observés à Yébouékro (Djékanou) dans le centre-sud de la Côte d'Ivoire
}

\author{
Kouadio Emile YOBOUE ${ }^{1 *}$, Conand Honoré KOUAKOU ${ }^{2}$, Odi Faustin AKOTTO ${ }^{1}$ et \\ Albert YAO-KOUAME ${ }^{1}$ \\ ${ }^{I}$ UFR STRM, Département des Sciences du Sol, Université Félix Houphouët-Boigny d'Abidjan Cocody, \\ Côte d'Ivoire, 22 BP. 582 Abidjan 22, Côte d'Ivoire. \\ ${ }^{2}$ UFR STRM, Laboratoire de Géomatériaux et Technologie de l'Habitat, Université Félix Houphouët-Boigny \\ d'Abidjan Cocody, Côte d'Ivoire, 22 BP. 582 Abidjan 22, Côte d'Ivoire. \\ "Auteur correspondant, E-mail: emileyoboue1@ gmail.com, Tel : +22507358768
}

\section{RESUME}

Le cuirassement observé dans des sols à plinthites suscite de nombreuses interrogations au sujet de leur mise en place, notamment les évolutions des oxydes. C'est pour répondre à cette préoccupation que la présente étude se fixe pour objectif de comprendre la dynamique des oxydes de silicium $\left(\mathrm{SiO}_{2}\right)$, d'aluminium $\left(\mathrm{Al}_{2} \mathrm{O}_{3}\right)$, de fer $\left(\mathrm{Fe}_{2} \mathrm{O}_{3}\right)$, de titane $\left(\mathrm{TiO}_{2}\right)$ et du manganèse $(\mathrm{MnO})$ dans ces sols lors des processus pédogénétiques. Pour y parvenir, un total de dix échantillons de sol, de roche et de cuirasse ont été soumis soit à une étude au microscope optique en lumière naturelle (LN) et en lumière polarisée (LP), soit à une étude des éléments totaux d'oxyde par Fluorescence à Rayons X (XRF) soit encore à une détermination de pH. Les résultats obtenus révèlent que les roches observées sur le terrain sont des brèches pyroclastiques basiques composées essentiellement de pyroxène, de plagioclase et d'épidote. Au niveau des deux types de sols étudiés, tandis que les teneurs en silice baissent de la roche au sol et à la cuirasse, les teneurs en oxyde de fer, quant à elles, augmentent. Alors qu'au niveau des teneurs en oxyde de titane les valeurs sont plus élevées dans les sols que dans la roche et dans la cuirasse. Cependant, des particularités existent au niveau de chaque type de sol. Ainsi, sur les cambisols, les sols sont plus riches en oxyde de silicium $(30,85 \%)$, d'aluminium $(41,85 \%)$, de manganèse $(33,82 \%)$ et de titane $(50,88 \%)$ que les cuirasses, qui elles, sont plus riches en oxydes de fer $(45,78 \%)$. Les teneurs en silice et en oxyde de manganèse sont plus faibles dans la cuirasse que dans le sol et dans la roche, tandis que les teneurs en alumine $(41,85 \%)$ et en titane $(50,88 \%)$ sont plus élevées dans les sols. Au niveau des andosols, les cuirasses sont plus riches en alumine $(38,68 \%)$ et en oxyde de fer $(46,94 \%)$ tandis que les sols sont plus riches en manganèse $(59,09 \%)$ et en titane $(46,79 \%)$. Pour chaque type de sol, les valeurs calculées des rapports caractéristiques $\left(\mathrm{SiO}_{2} / \mathrm{Al}_{2} \mathrm{O}_{3}\right)$ permettent de soupçonner des minéraux argileux de type 1/1, de la famille des kaolinites, dans les sols et dans les cuirasses. Ces minéraux argileux sont prédominants sur les oxy-hydroxydes de fer et d'aluminium dans la fraction argileuse de ces sols à caractère acide. En définitif, au cours de l'évolution de ces sols, les différents oxydes subissent de fortes variations de teneurs et s'accumulent soit dans le sol soit dans la cuirasse.

() 2019 International Formulae Group. All rights reserved

Mots clés: Sol à plinthites, oxyde, cambisol, andosol. 


\title{
Study of changes in the levels of a few oxides of two types of plinthite soil observed in Yebouékro (Djékanou) in south-central Côte d 'Ivoire
}

\begin{abstract}
The hardened grounds observed in plinthite soils raises many questions about their formation process, including variations in oxides. To address this concern, this study aims to understand the dynamics of silica $\left(\mathrm{SiO}_{2}\right)$, alumina $\left(\mathrm{Al}_{2} \mathrm{O}_{3}\right)$, iron $\left(\mathrm{Fe}_{2} \mathrm{O}_{3}\right)$, titanium $\left(\mathrm{TiO}_{2}\right)$ and manganese $(\mathrm{Mno})$ in these soils during pedogenetic processes. To achieve this, a total of ten sample of soil, rock and cuirasse were submitted either to a natural light microscope study (LN) and polarized light (LP), or to a study of the total elements of X-ray oxide (XRF) or to a $\mathrm{pH}$ determination. The results revealed that the rocks observed on the ground are basic pyroclastic holes composed mainly of pyroxene, plagioclase, and epidote. In the two types of soil studied, while silica levels decreased from rock to soil and cuirasse, iron levels increased. Whereas values in titanium levels are higher in soil than in the rock and in the cuirasse. However, specific features exist at the level of each soil type. Thus, on cambisols, soils are higher in silicon oxide $(30.85 \%)$, aluminum $(41.85 \%)$, manganese $(33.82 \%)$ and titanium $(50.88 \%)$ than cuirasses, which are more oxidized iron $(45.78 \%)$. Silica and manganese levels were lower in the cuirasse than in the soil and rock, while alumina levels $(41.85 \%)$ and titanium $(50.88 \%)$ were higher in soils. At the andosol level, cuirasses were higher in alumina $(38.68 \%)$ and iron oxide $(46.94 \%)$, while soils were higher in manganese $(59.09 \%)$ and titanium $(46.79 \%)$. For each soil type, the calculated values of the characteristic ratios $\left(\mathrm{SiO}_{2} / \mathrm{Al}_{2} \mathrm{O}_{3}\right)$ allow for the suspicion of $1 / 1$ clay type, such as kaolinite family, in soils and cuirasses. These clay minerals are predominant on iron and aluminum oxy-hydroxides in the clayey fraction of these acidic soils. In the final stages, during the evolution of these soils, the different oxides undergo large variations of levels and accumulate either in the soil or in the cuirasses.
\end{abstract}

(C) 2019 International Formulae Group. All rights reserved

Keywords: Plinthites soils, oxide, cambisol, andosol.

\section{INTRODUCTION}

Les sols à plinthites regroupent de grandes familles de sols qui se forment dans les régions tropicales où les saisons sèches et humides alternent (Bohi, 2010). Ces sols sont riches en éléments grossiers et possèdent un horizon B riche en argile. A faible profondeur, ces sols deviennent rouge tacheté et sont riches en fer. Ils deviennent immédiatement durs lorsqu'ils sont alternativement mouillés et séchés à plusieurs reprises formant alors une cuirasse (Foucault et Raoult, 2000).

Dans la zone de Djékanou, s'observent des sols présentant un aspect plinthique qui laissent transparaitre par endroit des dalles ou des blocs de cuirasses de dimensions et de consistances variables. L'observation de profils de sols révèle des cambisols et des andosols, plus ou moins profonds, très riches en éléments grossiers mais souvent à texture essentiellement argileuse dont l'ensemble donne à ces sols une coloration variable allant du brun (7,5YR) au brun grisâtre (2,5YR et 5YR).

Dans les sols, il est connu que le cuirassement est dû à une consolidation des oxydes du sol et, qu'il est lié essentiellement au dynamisme du fer et du manganèse (Assa, 2005 ; Bohi, 2010). Par ailleurs, ce sont les minéraux des roches qui s'altèrent et donnent les minéraux des sols (Benali, 2013). Dès lors, la question qui se pose est de comprendre comment les divers éléments chimiques ont évolué depuis l'altération de la roche-mère géologique jusqu'à la cuirasse dans le cas des sols de Yébouékro. C'est-à-dire, qu'elle est la nature des évolutions pédogéochimiques qui prévalent dans ces sols au cours de leur évolution pédogénétique aboutissant à leurs caractères plinthiques.

Le présent travail s'évertue à expliquer la dynamique des éléments chimiques susceptibles de renseigner sur les transformations minéralogiques de la roche- 
mère géologique aux cuirasses latéritiques. Dans ce contexte, il s'agira de :

- faire une étude pétrographique des roches affleurant sur ces sols ;

- examiner la dynamique de la silice $\left(\mathrm{SiO}_{2}\right)$, de l'alumine $\left(\mathrm{Al}_{2} \mathrm{O}_{3}\right)$, du fer $\left(\mathrm{Fe}_{2} \mathrm{O}_{3}\right)$, du titane $\left(\mathrm{TiO}_{2}\right)$ et du manganèse $(\mathrm{MnO})$ de la roche au sol et à la cuirasse le long d'une toposéquence.

\section{MATERIEL ET METHODES}

\section{Localisation de l'étude}

Les travaux se sont déroulés dans la région du Bélier, dans la sous-préfecture de Djékanou, précisément dans le village de Yébouékro, repéré par les coordonnées géographiques $\mathrm{X}=0266438$ et $\mathrm{Y}=0721298$ et située à 185 mètres d'altitude au-dessus du niveau de la mer. Le site d'étude est situé à 10 $\mathrm{km}$ de Toumodi et à $6 \mathrm{Km}$ de Djékanou sur l'axe Toumodi-Oumé (Figure 1).

\section{Milieu naturel de l'étude}

Le relief de la zone d'étude est vallonné et pittoresque et est dominé par des chaines de collines. Le réseau hydrographique du département de Djékanou est partagé entre deux grands bassins versants: le bassin versant du fleuve Bandama et celui du fleuve N'zi. (Yao et al., 2010). C'est un environnement soumis aux climats tropical et subtropical chaud, sec, et ensoleillé toute l'année avec deux saisons pluvieuses : une grande de mars à juin, une petite de septembre à octobre qui alternent avec deux saisons sèches (climat-data.org, 2016). La végétation de Djékanou est composée de savane arborée, de savane herbeuse et de forêts galeries (forêt de galeries mésophiles le long des cours d'eau) caractéristiques du "V Baoulé" (Tra Bi et al., 2015 ; Kouassi et al., 2016). Selon les observations faites par Yao-Kouamé et al. (2011), Kouakou et al. (2013), Yoboué et al.
(2018), les sols de la région sont des ferralsols, des gleysols et des cambisols.

\section{Méthodologie de travail}

L'étude a porté sur un total de 14 échantillons répartis comme suit :

- dix échantillons de sol issus des différents profils des cambisols et des andosols;

- deux échantillons de roche et ;

- deux échantillons composites de cuirasses prélevées respectivement sur le cambisol et sur l'andosol observés.

Le prélèvement des échantillons de sol s'est fait par profil de sol depuis l'horizon de profondeur jusqu'à l'horizon de surface de sorte à obtenir un échantillon par profil de sol. Des lames minces ont été taillées des échantillons de roches prélevées sur le terrain et décrites à l'aide d'un microscope polarisant en lumière naturelle (LN) et en lumière polarisée (LP) en vue d'une meilleure caractérisation de la roche.

Pour l'étude des éléments totaux d'oxyde, les échantillons de roche et de sol ont subi un concassage suivi d'un broyage et d'une pulvérisation pour atteindre une maille des grains de $75 \mu \mathrm{m}$ à $80 \%$. La pulpe a été mise en pastille pour la détermination des éléments chimiques (oxydes majeurs) par Fluorescence à Rayons X (XRF).

Le $\mathrm{pH}$ des sols a été déterminé par la méthode électrométrique, dans un rapport sol/solution de $1 / 2,5$. Puis, la différence $\left(\Delta \mathrm{pH}=\mathrm{pH}_{\text {eau }}-\mathrm{pH}_{\mathrm{Kcl}}\right)$ a été calculée en vue de l'appréciation de l'abondance relative des aluminosilicates par rapport aux oxyhydroxydes de fer et d'aluminium des sols selon Cruz et Camapum (2004).

Les données obtenues ont servi à l'élaboration des histogrammes et des courbes sur EXCEL et, au calcul des rapports caractéristiques $\left(\mathrm{SiO}_{2} \quad / \mathrm{Al}_{2} \mathrm{O}_{3}\right)$ et $\left(\mathrm{SiO}_{2} /\right.$ $\left.\left(\mathrm{Fe}_{2} \mathrm{O}_{3}+\mathrm{Al}_{2} \mathrm{O}_{3}\right)\right)$ en tenant compte des différentes unités de sol en vue de leur interprétation. 


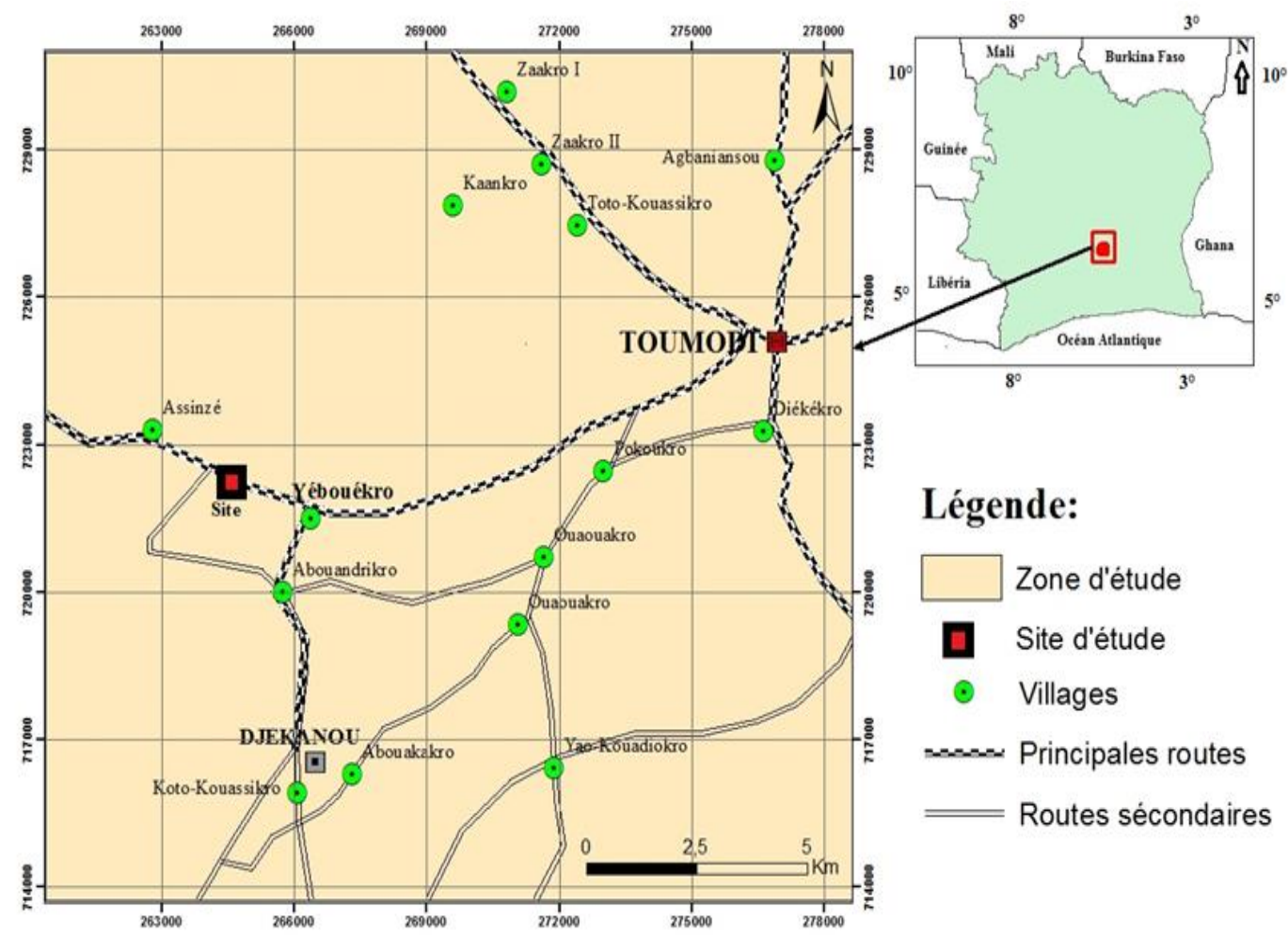

Figure 1: Carte de localisation du site d'étude.

\section{RESULTATS}

Nature pétrographique de la roche observée

L'étude des caractères pétrographiques des formations rocheuses, observées sur le terrain, s'est avérée nécessaire pour établir d'éventuels liens entre la minéralogie de la roche-mère géologique et celle du sol qui dérive de son altération. Du point de vue macroscopique, les formations étaient des roches volcaniques pyroclastiques dans lesquelles s'observaient des clastes prises dans un ciment pour constituer en tout la roche. C'était des brèches volcaniques à caractère tufacé. Ces roches étaient gris verdâtres, avec des minéraux sombres de taille inférieure à $2 \mathrm{~mm}$ (Figure 2).

$\mathrm{Au}$ microscope, les roches montraient un caractère amphibolique avec des phénocristaux de pyroxène maclé (Figure 3 ). C'était des pyroclastites basiques présentant des compositions minéralogiques variées. Ces minéraux étaient soit des minéraux ferromagnésiens ou riches en fer, soit des minéraux alumineux, soit des minéraux de la silice ou soit encore des opaques.

Des minéraux ferromagnésiens ou riches en fer

Il s'agissait du pyroxène et de l'épidote dont les formules sont les suivantes :

-le pyroxène : $\mathrm{X}_{1-\mathrm{P}} \mathrm{Y}_{1+\mathrm{P}} \mathrm{Z}_{2} \mathrm{O}_{6}$ avec $0<\mathrm{P}<1$ où :

$\mathrm{X}=\mathrm{Ca}, \mathrm{Na}$;

$\mathrm{Y}=\mathrm{Mg}, \mathrm{Fe}^{2+}, \mathrm{Mn}, \mathrm{Li}, \mathrm{Ni}, \mathrm{Al}, \mathrm{Fe}^{3+}, \mathrm{Cr}$, Ti et

$\mathrm{Z}=\mathrm{Si}, \mathrm{Al}$

- les épidotes $\left(\left(\mathrm{Si}_{2} \mathrm{O}_{7} / \mathrm{SiO}_{4} / \mathrm{OH} / \mathrm{O}\right) \mathrm{Ca}_{2} \mathrm{Al}_{2}\right.$ $\left.\left(\mathrm{Fe}^{3+}, \mathrm{Al}\right)\right)$;

\section{Des minéraux alumineux}

Ici, les minéraux contenant de l'aluminium étaient les plagioclases $(\mathrm{Ca}, \mathrm{Na}$ $\left.\left(\mathrm{Al}_{1,2} \mathrm{Si}{ }_{2,3} \mathrm{O}_{8}\right)\right)$, mais aussi les épidotes $\left(\left(\mathrm{Si}_{2} \mathrm{O}_{7} / \mathrm{SiO}_{4} / \mathrm{OH} / \mathrm{O}\right) \mathrm{Ca}_{2} \mathrm{Al}_{2}\left(\mathrm{Fe}^{3+}, \mathrm{Al}\right)\right)$;

\section{Des minéraux de la silice}

Les minéraux de la silice observés étaient surtout le quartz $\left(\mathrm{SiO}_{2}\right)$.

Des minéraux opaques (oxydes)

Ce sont des minéraux qui ne se 
laissaient pas traverser par la lumière et qui n'ont pu être décrits en lames minces.

\section{Dynamique des oxydes de la roche au sol et à la cuirasse \\ Au niveau des cambisols}

La Figure 4 traduit les évolutions des oxydes de silicium, d'aluminium, de fer, de manganèse et de titane de la roche saine au sol et à la cuirasse au niveau des cambisols. L'observation de cette figure permet de constater que les teneurs de ces différents éléments étaient très variables.

Les teneurs en silice $\left(\mathrm{SiO}_{2}\right)$ diminuaient de la roche saine $(48,84 \%)$ au sol $(30,85 \%)$ et à la cuirasse $(20,29 \%)$ comme s'il $\mathrm{y}$ avait une perte de silice de la roche saine à la cuirasse. Cependant, ce constat peut aussi être dû à la concentration d'autres éléments dans le sol et dans la cuirasse.

L'alumine $\left(\mathrm{Al}_{2} \mathrm{O}_{3}\right)$ augmentait de la roche au sol et diminuait dans la cuirasse en passant respectivement de $24,94 \%$ à $41,85 \%$ et à 33,20\%. L'oxyde d'aluminium issu de l'altération des minéraux alumineux comme les plagioclases était plus concentré dans le sol que dans la cuirasse.

L'oxyde de fer $\left(\mathrm{Fe}_{2} \mathrm{O}_{3}\right)$ présentait une teneur croissante de la roche au sol et à la cuirasse. Les teneurs passaient de 15,14\% dans la roche à $39,06 \%$ dans le cambisol et à $45,78 \%$ dans la cuirasse, donnant l'idée d'une évacuation des éléments ferrugineux contenus dans la roche et dans le sol et à leur accumulation dans la cuirasse. Ce fer proviendrait des pyroxènes et des épidotes contenues dans la roche saine observée sur le terrain.

Comme le silicium, les teneurs en oxydes de manganèse $(\mathrm{MnO})$ diminuaient de la roche $(42,64 \%)$ au sol $(33,82 \%)$ et à la cuirasse $(23,52 \%)$. Les cuirasses au niveau des cambisols étaient donc moins manganésifères que la roche.

L'oxyde de titane $\left(\mathrm{TiO}_{2}\right)$ était plus concentré dans le sol à $50,88 \%$. Le $\mathrm{TiO}_{2}$ pourrait provenir de certains pyroxènes riches en titane, mais aussi de l'anatase ou du rutile qui eux sont des oxydes, opaques au microscope optique. Lors de l'altération des roches, ces minéraux libéraient dans le sol l'oxyde de titane dont la migration n'était pas encore effective au niveau de la cuirasse.

\section{Au niveau des andosols}

La Figure 5 permet de mettre en évidence les différentes variations de teneurs des oxydes de la roche au sol et à la cuirasse au niveau des andosols observés.

La dynamique de la silice $\left(\mathrm{SiO}_{2}\right)$ était identique à celle observée au niveau des cambisols. Les teneurs de silice diminuaient de la roche au sol et à la cuirasse. Les teneurs variaient de $46,37 \%$ dans la roche à $34,35 \%$ dans le sol et à 19,26\% dans la cuirasse. Ce qui permet de comprendre que les cuirasses issues de l'évolution pédogénétique dans ces sols ne sont pas dues à une prise en masse d'éléments quartzifères. En fait, les roches ne sont pas aussi riches en quartz pour le libérer dans le sol au cours de l'altération. La silice de la roche était en grande partie due à celle des silicates qu'elle contenait.

Les teneurs en alumine $\left(\mathrm{Al}_{2} \mathrm{O}_{3}\right)$ augmentaient de la roche au sol et à la cuirasse en passant de $29,07 \%$ dans la roche à $32,24 \%$ dans le sol et $38,68 \%$ dans la cuirasse. Il y avait donc eu une concentration de l'alumine dans la cuirasse que dans le sol au niveau des andosols.

$\mathrm{La}$ dynamique du fer $\left(\mathrm{Fe}_{2} \mathrm{O}_{3}\right)$ était similaire à cette de l'alumine. Les teneurs croissaient de la roche au sol et à la cuirasse avec des teneurs de $15,52 \%$ dans la roche, $37,52 \%$ dans le sol et $46,94 \%$ dans la cuirasse. Les cuirasses des andosols étaient donc non seulement alumineuses mais bien aussi ferrugineuses comme cela s'observait au niveau des cambisols.

L'oxyde de manganèse $(\mathrm{MnO})$ présentait des teneurs plus élevées dans le sol $(59,09 \%)$ que dans la roche $(26,36 \%)$ et dans la cuirasse $(14,54 \%)$. Les cuirasses des andosols seraient donc moins concentrées en oxydes de manganèse comme cela s'observait aussi au niveau des cambisols.

Le titane $\left(\mathrm{TiO}_{2}\right)$ présentait une dynamique comparable à celle observée au niveau des cambisols avec des teneurs plus élevées dans le sol $(46,79 \%)$. 


\section{Rapports caractéristiques Silice/Alumine, Silice/sesquioxydes}

Les résultats obtenus, suite à l'analyse de la silice $\left(\mathrm{SiO}_{2}\right)$ et des sesquioxydes $\left(\mathrm{Fe}_{2} \mathrm{O}_{3}\right.$ et $\mathrm{Al}_{2} \mathrm{O}_{3}$ ), ont permis le calcul des rapports caractéristiques suivants :

$-\mathrm{SiO}_{2} / \mathrm{Al}_{2} \mathrm{O}_{3}$

$-\mathrm{SiO}_{2} /\left(\mathrm{Fe}_{2} \mathrm{O}_{3}+\mathrm{Al}_{2} \mathrm{O}_{3}\right)$.

Ces rapports sont importants dans la mesure où ils permettent d'apprécier, non seulement, le niveau d'évolution du sol, mais aussi le type de minéral argileux présent dans le sol ainsi que les niveaux à concrétionnement ou à cuirassement dans les profils de sol. Les résultats exprimés à travers les Figures 6 et 7 ont permis de vérifier la grande variabilité de ces rapports sur chacun des échantillons étudiés. Pour les résultats obtenus, les valeurs les plus élevées des rapports $\mathrm{SiO}_{2} / \mathrm{Al}_{2} \mathrm{O}_{3}$ étaient voisines de 2 pour les roches tandis qu'elles étaient voisines de 1 au niveau des sols et des cuirasses.

$\begin{array}{ccc}\mathrm{Le} & \text { rapport } & \text { moléculaire } \\ \mathrm{SiO}_{2} /\left(\mathrm{Al}_{2} \mathrm{O}_{3}+\mathrm{F}_{2} \mathrm{O}_{3}\right) & \text { suivait, } & \text { dans les }\end{array}$

échantillons, une courbe grossièrement parallèle à celle du rapport précédant $\mathrm{SiO}_{2} / \mathrm{Al}_{2} \mathrm{O}_{3}$. Cependant, au niveau des andosols, la courbe du rapport $\mathrm{SiO}_{2} /\left(\mathrm{Al}_{2} \mathrm{O}_{3}+\mathrm{F}_{2} \mathrm{O}_{3}\right)$ se rapprochait sensiblement de celle du rapport $\mathrm{SiO}_{2} / \mathrm{Al}_{2} \mathrm{O}_{3}$ dans les cuirasses.

\section{Valeurs de pH mesuré}

Les valeurs déterminées de $\mathrm{pH}$ sont présentées dans le Tableau 1. L'observation du tableau permet de constater que les valeurs du $\mathrm{pH}$ variaient de 4,8 à 6,08 . Ces valeurs sont caractéristiques des sols acides à moyennement acides. Cependant, il convient de noter que les cambisols révélaient un caractère plus acide avec des valeurs de $\mathrm{pH}$ allant de 4,8 à 5,2 dans les sols et de 5,3 à 5,38 dans les cuirasses. Alors que, dans les andosols, les pH variaient de 5,06 à 6,08 dans les sols et de 5,77 à 5,94 dans les cuirasses. La variation $\mathrm{du} \mathrm{pH}(\Delta \mathrm{pH}=\mathrm{pHeau}-\mathrm{pHkcl})$ donnait toujours des valeurs positives allant de 0,08 à 1,02 .

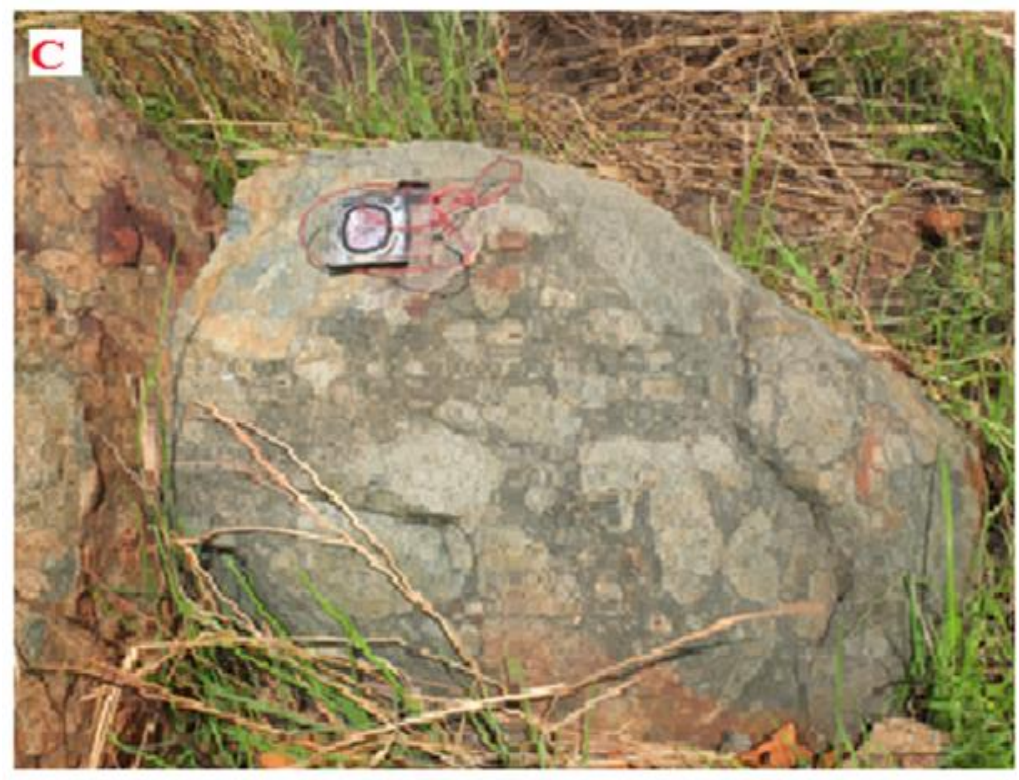

Figure 2 : Affleurement rocheux observé à Yébouékro. 


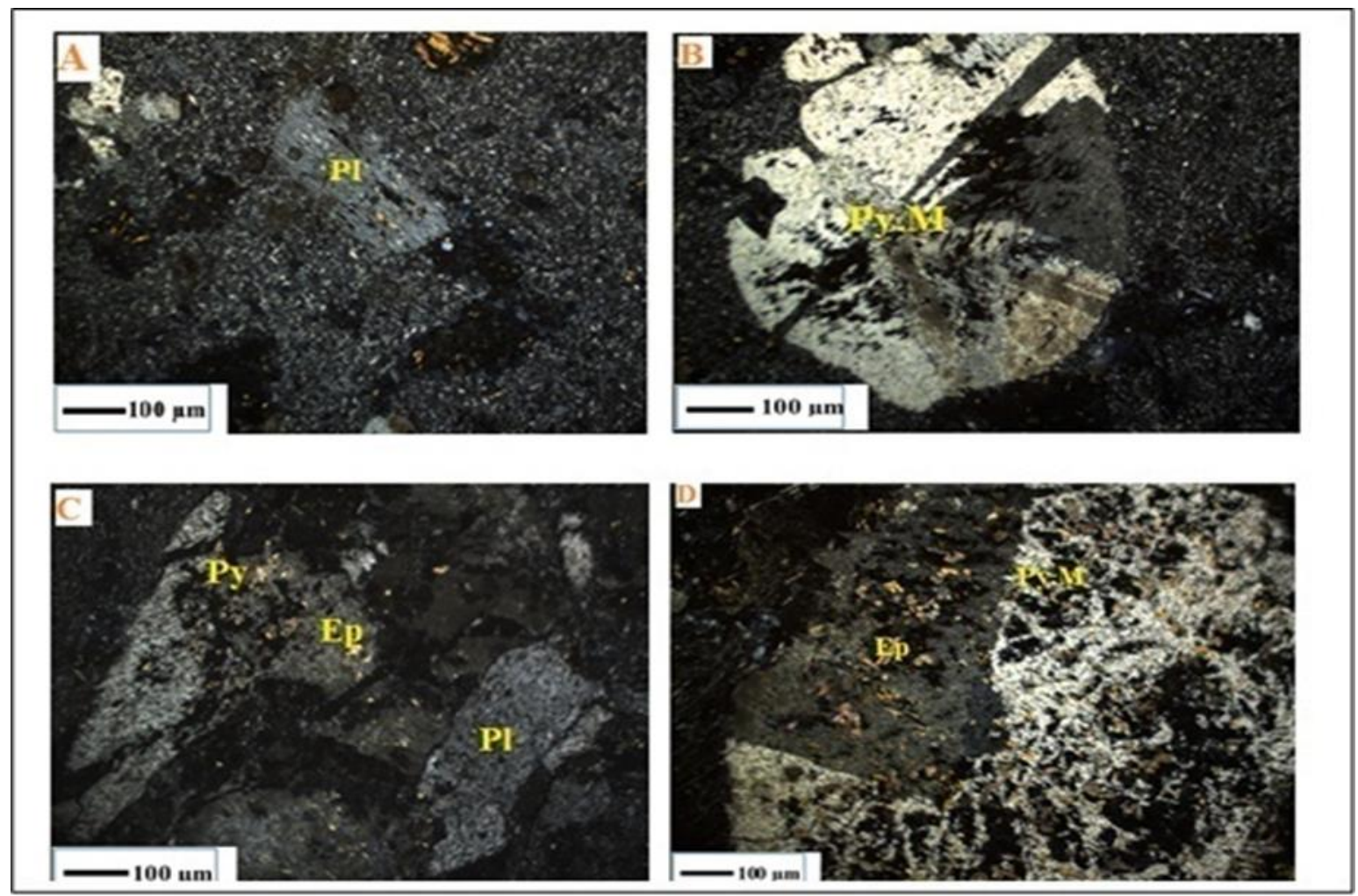

A et C : Minéraux observés dans le ciment pyroclastique; B et D: Minéraux observés dans les clastes pyroclastiques. $\mathrm{Pl}$ : Plagioclase ; Py.M : Pyroxène mâclé; Py : Pyroxène ; Ep : Epidote.

Figure 3 : Observation des roches au microscope.

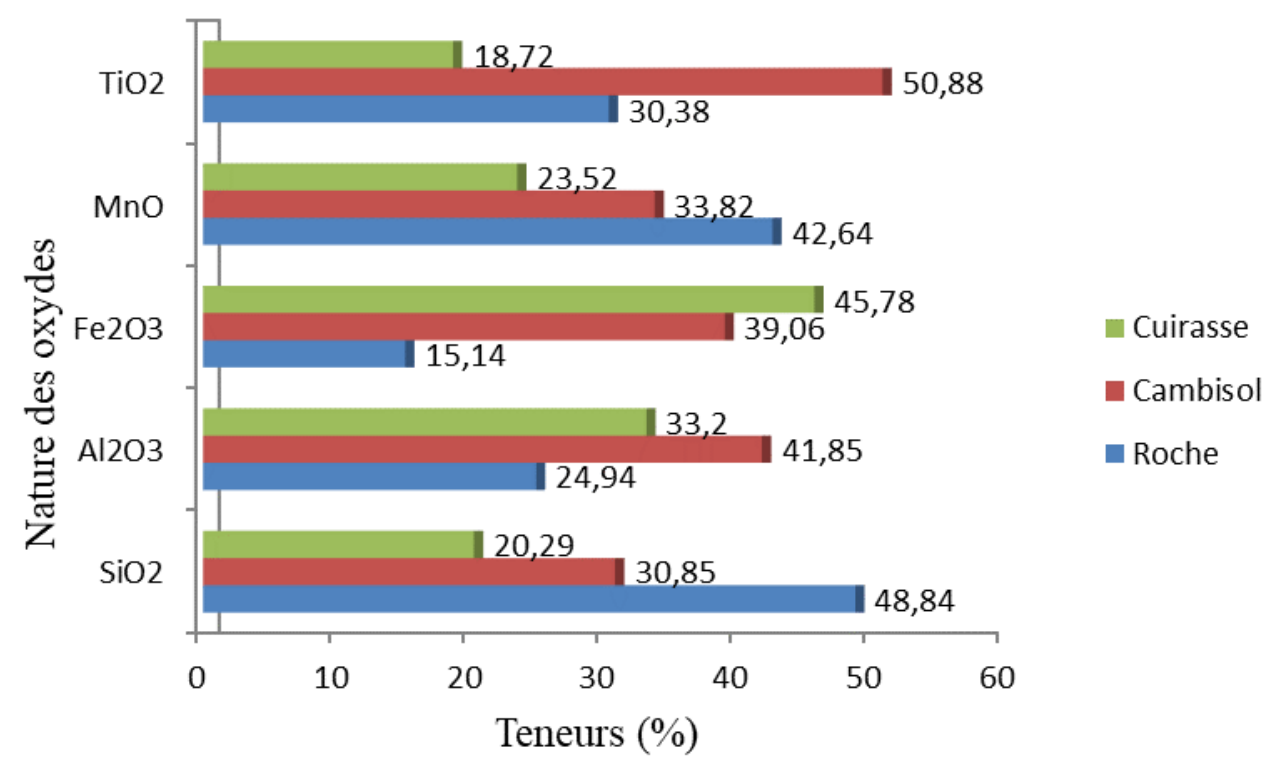

Figure 4 : Evolution des oxydes de la roche au sol et à la cuirasse au niveau des cambisols de Yébouékro. 


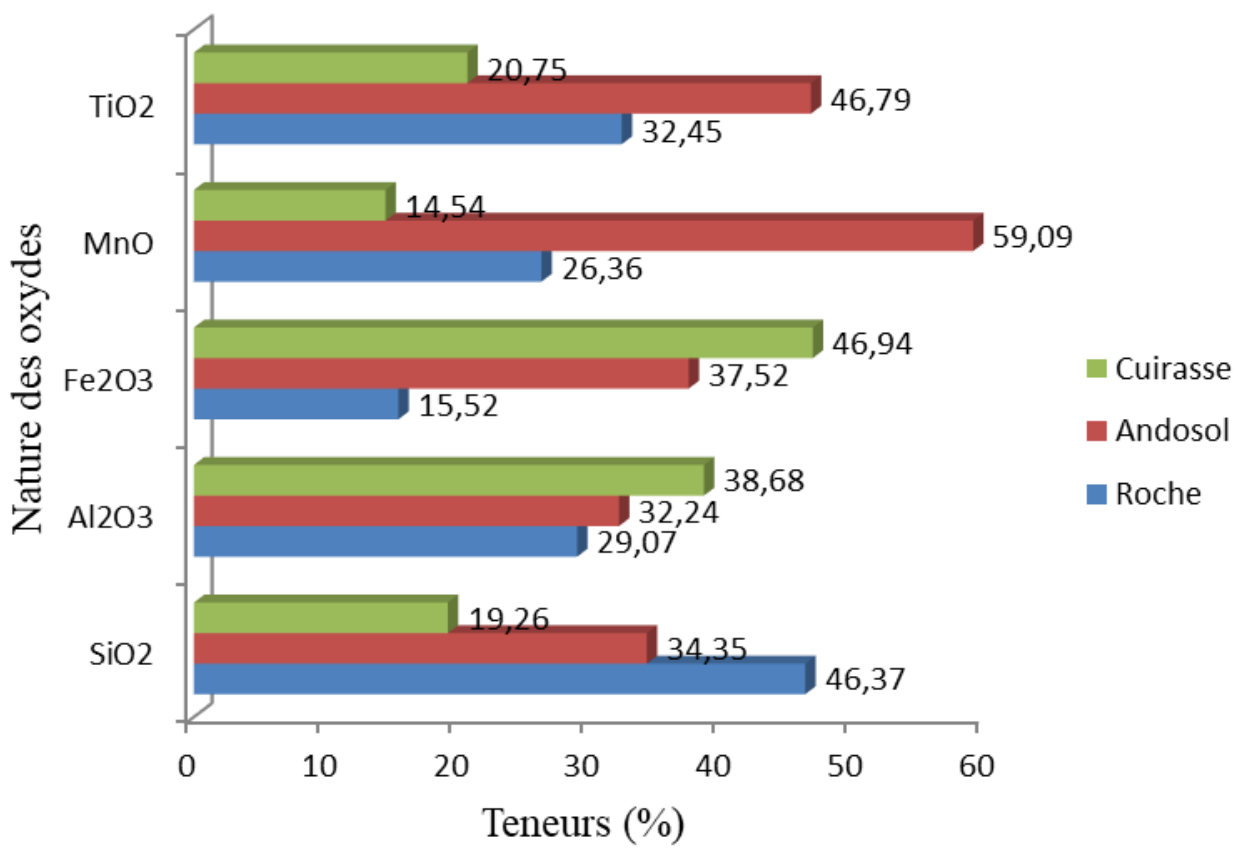

Figure 5 : Evolution des oxydes de la roche au sol et à la cuirasse au niveau des andosols de Yébouékro.

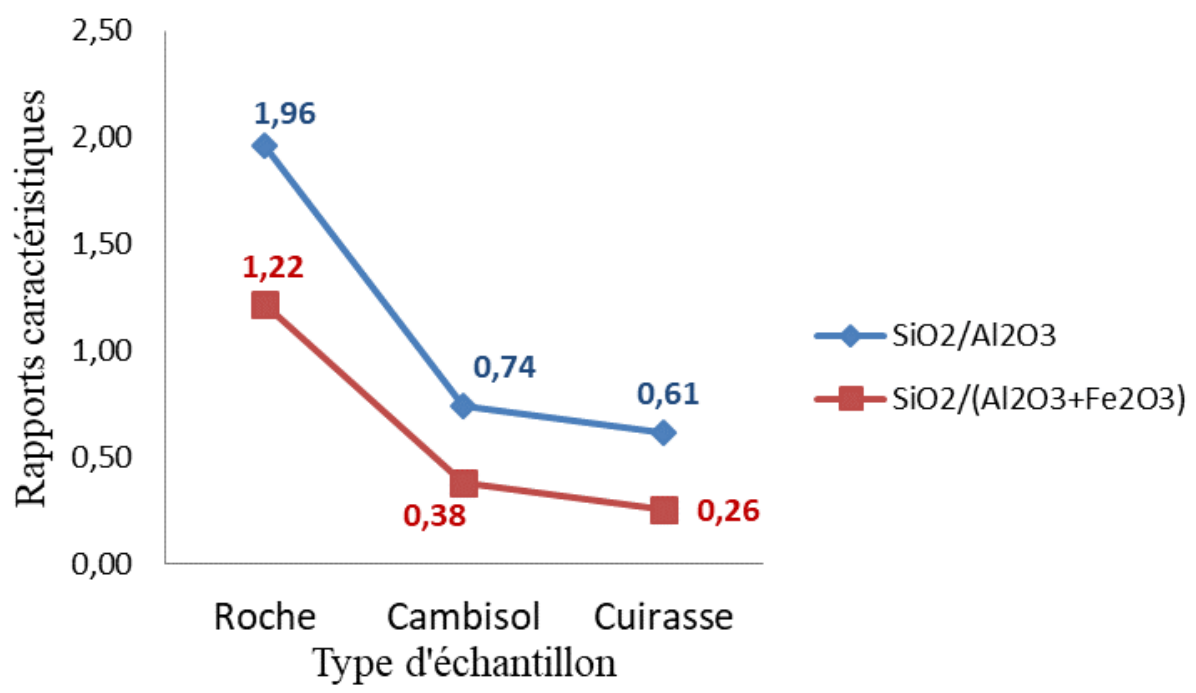

Figure 6: Courbes de variation des rapports caractéristiques $\mathrm{SiO}_{2} / \mathrm{Al}_{2} \mathrm{O}_{3}$ et $\mathrm{SiO}_{2} /\left(\mathrm{Al}_{2} \mathrm{O}_{3}+\mathrm{F}_{2} \mathrm{O}_{3}\right)$ au niveau des cambisols. 


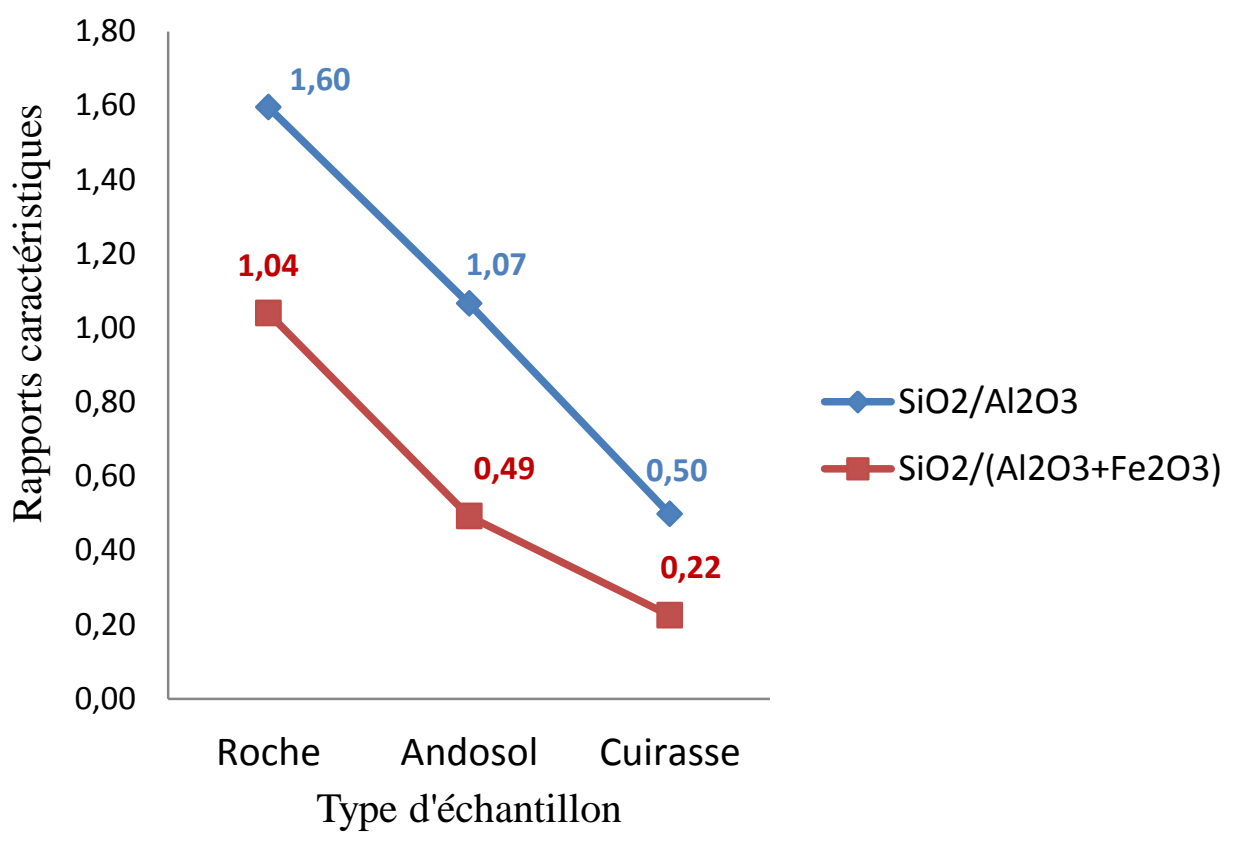

Figure 7: Courbes de variation des rapports caractéristiques $\mathrm{SiO}_{2} / \mathrm{Al}_{2} \mathrm{O}_{3}$ et $\mathrm{SiO}_{2} /\left(\mathrm{Al}_{2} \mathrm{O}_{3}+\mathrm{F}_{2} \mathrm{O}_{3}\right)$ au niveau des andosols.

Tableau 1: Valeurs du $\mathrm{pH}_{\text {eau }}$, du $\mathrm{pH}_{\mathrm{KCl}}$ et du $\Delta \mathrm{pH}$ en fonction du type de sols.

\begin{tabular}{llll}
\hline Types de sols & pHeau & pHkcl & $\Delta \mathbf{p H}(\mathbf{p H e a u}-\mathbf{p H k c l})$ \\
\hline Cambisol & 5,2 & 4,8 & 0,4 \\
Cuirasse sur cambisol & 5,38 & 5,3 & 0,08 \\
Andosol & 6,08 & 5,06 & 1,02 \\
Cuirasse sur andosol & 5,94 & 5,77 & 0,17 \\
\hline
\end{tabular}

\section{DISCUSSION}

\section{Nature de la roche}

L'observation macroscopique sur le terrain montre qu'il s'agit de roches volcaniques, essentiellement des pyroclastites, constituées des clastes prises dans un ciment, le tout constituant la roche. Ce sont des brèches volcaniques à caractère tufacé comme l'observait Yoboué (2010) sur des sols brunifiés à Kahankro. Ces roches sont caractérisées par une minéralogie très variée et s'altèrent en donnant les sols plinthiques observés dans la zone.

$\mathrm{Au}$ niveau microscopique, les études permettent de comprendre que du point de vue minéralogique, la roche mère pyroclastite observée est composée essentiellement de pyroxène, de plagioclase et d'épidote. Sous l'action de l'altération hydrolytique, ces minéraux libèrent dans le sol leurs divers éléments qui sont soit ferrugineux, alumineux, manganésifères, titanifères, ou siliceux. Ceuxci sont, soit évacués par les eaux de drainage, soit recombinés pour former les minéraux 
secondaires de néogenèse comme montré par certains travaux (Krekeler et al., 2005 ; Ferry et al., 2003).

Les minéraux opaques observés en lames minces sont supposés être la pyrite $\left(\mathrm{FeS}_{2}\right)$ et la chalcopyrite $\left(\mathrm{CuFeS}_{2}\right)$ comme soupçonné par les travaux de Yao-kouamé (2007) dans le village voisin de Kanhankro dans des formations pyroclastiques et des roches vertes.

\section{Dynamique des oxydes de la roche au sol et à la cuirasse}

Les résultats des analyses pédogéochimiques montrent que les cambisols observés à Yébouékro sont plus riches en oxyde de silicium, d'aluminium, de manganèse et de titane que les cuirasses observées dans ces sols. Seul l'oxyde de fer semble être plus concentré dans la cuirasse avec $45,78 \%$. Les cuirasses observées au niveau des cambisols de Yébouékro sont donc plus ferrugineuses que alumineuses, manganésifères ou titanifères. Cependant, il s'observe aussi quelques similarités au niveau de la dynamique de certains éléments. Ainsi, les évolutions de la silice sont semblables à celles du manganèse dont les teneurs diminuent potentiellement de la roche au sol et à la cuirasse. De même, les teneurs en alumine $(41,85 \%)$ et en titane $(50,88 \%)$ sont plus élevées dans les sols.

$\mathrm{Au}$ niveau des andosols, le constat général est tel que les cuirasses sont plus riches en alumine $(38,68 \%)$ et en oxyde de fer $(46,94 \%)$ tandis que les sols sont plus riches en manganèse $(59,09 \%)$ et en titane $(46,79 \%)$.

Par ailleurs, des similarités peuvent s'observer au niveau de la dynamique de certains éléments dans les deux types de sol. Il s'agit des teneurs en silice qui diminuent de la roche au sol et à la cuirasse, des teneurs en fer qui augmentent de la roche au sol et à la cuirasse et des teneurs en titane qui sont plus élevées dans les sols que dans la roche et dans la cuirasse.

Ces différents oxydes sont donc à teneurs variables quel que soit le type de sol comme montré par Legros (2013). De plus, ces teneurs variables des différents éléments dans les sols et les cuirasses montrent que les teneurs des éléments chimiques, lors de l'altération, sont bien différents de leurs teneurs dans la roche originelle dont ils proviennent selon les travaux de Nono et al. (2009) et de Wyns et al. (2014). Ces variations de teneurs proviennent de l'altération des minéraux des roches observées et décrites sur le terrain et au laboratoire. Ainsi, la silice dans les sols proviendrait de l'altération des silicates observés en lames minces dans les roches. Il s'agit des plagioclases, des pyroxènes, des épidotes et surtout du quartz. L'alumine serait issue de l'altération de minéraux alumineux comme les plagioclases $\left(\mathrm{Ca}, \mathrm{Na}\left(\mathrm{Al}_{1,2} \mathrm{Si}_{2,3} \mathrm{O}_{8}\right)\right.$ ), et aussi de l'épidote $\left(\left(\mathrm{Si}_{2} \mathrm{O}_{7} / \mathrm{SiO}_{7} / \mathrm{OH} / \mathrm{O}\right) \mathrm{Ca}_{2} \mathrm{Al}_{2}\right.$ (Al, $\left.\mathrm{Fe}^{+}\right)$) qui contient de l'aluminium dans sa structure. Le fer proviendrait des minéraux ferromagnésiens, comme le pyroxène et aussi de l'épidote ( $\left(\mathrm{Si}_{2} \mathrm{O}_{7} / \mathrm{SiO}_{7} / \mathrm{OH} / \mathrm{O}\right) \mathrm{Ca}_{2} \mathrm{Al}_{2}(\mathrm{Al}$, $\left.\mathrm{Fe}^{+}\right)$) et de la pyrite $\left(\mathrm{FeS}_{2}\right)$. L'oxyde de manganèse dans les sols proviendrait de l'altération de certains pyroxènes qui contiendraient du Mn dans leur chimisme. Le $\mathrm{TiO}_{2}$ pourrait provenir de certains pyroxènes riches en titane selon la formule générale des pyroxènes, mais aussi de l'anatase ou du rutile qui eux sont des oxydes, opaques au microscope optique comme observé en lame mince. Au cours de l'altération, les hydrates de fer sont libérés et jalonnent les clivages et les flancs des cristaux. Il se forme une trame résistante, où l'on reconnait la structure du minéral, qui enserre des produits pulvérulents ferrugineux jaunes et rouges. Ces pseudomorphoses sont friables et se réduisent facilement en un mélange de produits alumineux imbibés d'hydroxydes de fer (Meunier, 2003). Ce mélange avec des produits ferrugineux associés à la matière organique conduit à la coloration brune observée et caractéristique des cambisols observés.

Les valeurs des rapports caractéristiques $\mathrm{SiO}_{2} / \mathrm{Al}_{2} \mathrm{O}_{3}$ sont voisines de 1 dans les sols tout comme dans les cuirasses. Ces valeurs montrent que les minéraux argileux des sols et des cuirasses seraient probablement des minéraux argileux de type 
1/1 de la famille des kaolinites. Ce qui serait en désaccord avec les travaux de Yoboué et al. (2014). Car sur des cambisols issus de formations tufacées à Kahankro et à Anikro, deux localités voisines de Yébouékro, ces auteurs identifient en plus de la kaolinite, l'illite et la chlorite. Cependant, cette minéralisation argileuse de type 1/1 permet de penser que le processus géochimique prévalant dans ces sols est la monosiallitisation.

$\mathrm{Au}$ niveau des andosols, la courbe du rapport $\mathrm{SiO}_{2} /\left(\mathrm{Al}_{2} \mathrm{O}_{3}+\mathrm{F}_{2} \mathrm{O}_{3}\right)$ se rapproche sensiblement de celle du rapport $\mathrm{SiO}_{2} / \mathrm{Al}_{2} \mathrm{O}_{3}$ dans les cuirasses. En fait, la teneur en silice est beaucoup trop faible par rapport à la teneur en sesquioxydes de fer et d'aluminium dans les cuirasses ; ce qui rapproche sensiblement les courbes des rapports caractéristiques.

Les andosols et les cambisols étudiés ont un $\mathrm{pH}$ acide comme les cambisols observés par Kfuban et al. (2014). Les différences de $\mathrm{pH}$ qui ont été calculées ont donné des valeurs positives. Cette variation de $\mathrm{pH}$ montre que l'acidité du sol n'est pas le fait des ions du fer, ni de l'aluminium ou du manganèse libérés dans le sol, mais, plutôt des ions $\mathrm{OH}^{-}$. De plus, ces valeurs calculées de $\Delta \mathrm{pH}(\Delta \mathrm{pH}=\mathrm{pHeau}-\mathrm{pHkcl})$, toujours positives, signifient, d'après les travaux de Cruz et Camapum (2004), que dans les cambisols et les andosols de Yébouékro, il y a une prédominance des minéraux argileux sur les oxy-hydroxydes de fer et d'aluminium dans la fraction argileuse des sols.

\section{Conclusion}

L'étude visant à la caractérisation de la dynamique des oxydes de fer, d'aluminium, de manganèse, de titane et de la silice a montré parfois des similarités ou des dispersions. Ainsi, dans les sols à plinthites de Yébouékro, au niveau des cambisols, la silice et le manganèse évoluaient de la même manière tout comme l'alumine et le titane. Alors qu'au niveau des andosols, c'était l'alumine et le fer d'une part, et le manganèse et le titane d'autre part, qui évoluaient de la même manière selon qu'on passait de la roche au sol et à la cuirasse. La silice, l'oxyde de fer et l'oxyde de titane présentaient la même dynamique dans les cambisols que dans les andosols. En gros, les minéraux des roches, riches en fer, en aluminium, en manganèse, en titane et en silicium, s'altérant, libèrent dans le milieu édaphique des éléments chimiques dont la dynamique est variable selon le processus pédogénétique prédominant.

\section{CONFLITS D'INTERETS}

Les auteurs déclarent n'avoir aucun conflit d'intérêt.

\section{CONTRIBUTIONS DES AUTEURS}

KEY, CHK et OFA ont participé à la réalisation des travaux sur le terrain et au laboratoire puis, à la conception, à la rédaction et à la mise en forme de l'article ; AYK étant le superviseur à la réalisation de toutes les étapes de ce travail.

\section{REMERCIEMENTS}

Nous tenons à remercier les autorités administratives de Djékanou et les autorités coutumières du village de Yébouékro pour le chaleureux accueil à nous réservé dans leur localité.

\section{REFERENCES}

Assa AD. 2005. Précis de pédologie. A l'usage des étudiants du premier cycle des études universitaires. Editions Sciences, EDUCI n ${ }^{\circ} 7226,94 \mathrm{p}$.

Benali M. 2013. Les altérations des granites et des basaltes. Mémoire Présenté pour l'obtention du Grade d'Ingéniorat d'Etat en Sciences de la Terre Option : Ensembles Cristallins. Université d'Oran Belgaid, $69 \quad$ p., http://www.academia.edu/4000210/Lalt \%C3\%A9ration_des_granites_et_des_ba saltes $(11 / 01 / 2018)$

Bohi ZPB. 2010. Caractérisation des sols latéritiques utilisés en construction routière : cas de la région de l'Agneby (Côte d'Ivoire). Mechanics of materials. Ecole des Ponts ParisTech, 142p, https://pastel.archives-ouvertes.fr/pastel00503010/document (07/11/2018) 
Ferry B, Freycon V, Paget D. 2003. Description et dynamique des milieux forestiers: Genèse et fonctionnement hydrique des sols sur socle cristallin en Guyane. Rev. For. Fr. $L V-\mathrm{n}^{\circ}$ spécial 2003 : 37-59.

https://www6.nancy.inra.fr/foret-boislerfob/Les-equipes/Ecologie-

Forestiere/Publications/Non-referenceesISI

Climat-data.org. 2016, https://fr.climatedata.org/region/1317/\#example2 (22/01/2018)

Cruz LM, Camapum de CJ. 2004. Etude géotechnique des processus érosifs dans le Centre Ouest du Brésil. XXIIe rencontre Universitaire de Génie Civil 2004-Ville et Génie Civil, 8 p.

Foucault A, Raoult JF. 2000. Dictionnaire de géologie, 5e édition Broché, 11 p., https://www.abebooks.fr/rechercherlivre/titre/dictionnaire/auteur/foucault/ (12/09/2016)

Kfuban YBP, Tiamgne YA, Assembo MCF, Ranst VE. 2014. Effet des trous de plantation sur les plants greffés de manguier (Mangifera indica L.) sur un Cambisol du Yongka Western Highlands Research Garden Park Nkwen-Bamenda au Cameroun. Int. J. Biol. Chem. Sci. 8(6): 2659-2668. www.ifgdg.org/index.php?option=com

Kouakou YKN, Yao GF, Nangah KY, Guety TP, Yao-Kouamé A. 2013. Diagnostic $\mathrm{du}$ fonds pédogéochimique des sols développés sur matériaux volcanosédimentaires au Blafo-Guéto (Toumodi) dans le Centre-Sud de la Côte d'Ivoire. International Journal of Innovation and Applied Studies, 2(3): 344-354. citeseerx.ist.psu.edu/viewdoc/download? doi $=10.1 .1 .303 .1479 \&$ rep $=$ rep $1 \&$ type $=p$ df

Kouassi AM, Ahoussi KE, Kouakou KE, Mamadou A, Biemi J. 2016. Analyse comparative entre la distribution des fréquences de conductivité électrique et les faciès géochimiques des eaux des aquifères de socle (Côte d'Ivoire). Int. J.
Biol. Chem. Sci., 10(1): 435-446. http://dx.doi.org/10.4314/ijbcs.v10i1.33

Krekeler M, Hammerly E, Rakovan J, Guggenheim S. 2005. Microscopie studies of the palygorskite to smectite transformation. Clays and Clay Minerals, 53(1): 92-99. DOI: https://doi.org/10.1346/CCMN.2005.053 0109

Legros J-P. 2013. Latérites et autres sols des régions intertropicales. Conférence $\mathrm{n}^{\circ} 4252$, Séance académique du 09/12/2013, Bulletin $\mathrm{n}^{\circ} 44$ : 369-382, http://www.ac-sciences-lettresmontpellier.fr/ (17/07/2018)

Meunier A. 2003. Argiles. Paris : GB éditions scientifiques, $433 \mathrm{p}$.

Nono A, Likeng JGH, Wabo H, Tabue Youmbi G, Biaya S. 2009. Influence de la nature lithologique et des structures géologiques sur la qualité et la dynamique des eaux souterraines dans les hauts plateaux de l'Ouest-Cameroun. Int. J. Biol. Chem. Sci., 3(2): 218-239. http://dx.doi.org/10.4314/ijbcs.v3i2.4451 6

Tra Bi ZA, Brou YT, Mahe G. 2015. Analyse par télédétection des conditions bioclimatiques de végétation dans la zone de contact forêt-savane de côte d'ivoire : cas du «V » baoulé. XXVIIIe Colloque de l'Association Internationale de Climatologie, Liège 2015.http://www.Climato.be/aic/colloqu es/actes/ACTES_AIC2015/1\%20Modeli sation\%20Cryosphere\%20et\%20Teledet ection/012-TRA_BI-078-083.pdf (09/03/2019)

Wyns R, Cornu S, Prognon C. 2014. Régolithe et sol : l'interface entre lithosphère et atmosphère. Geosciences, BRGM, 2014: 8-15, https://halbrgm.archives-ouvertes.fr/hal-01075128

Yao-kouamé A. 2007. Caractéristiques physiques des sols brunifiés dérivés des formations du complexe volcanosédimentaire de Kanhankro (Toumodi) en moyenne cote d'ivoire, Rev. CAMES - Série A, 05: 39-52. greenstone.lecames.org/collect/revu/inde 
x/assoc/HASHdddc.dir/A-005-00-076086.pdf.

Yao-Kouamé A, Koné B, Gala Bi TJ. 2011. Caractérisation morphopédologique des sols bruns eutrophes développés sur les complexes volcano-sédimentaires ouest africains du birimien à Blafo en Côte d'ivoire. Africa Geoscience Review, 18(1) : 23-32.

https://www.academia.edu/38006532/Ca racterisation_de_sol_bruns_eutrophe_de veloppe_sur_les_complexes_volcano_se dimentaires_en_Cute_d_Ivoire

Yao KA, Kouassi AM, Koffi YB, Biemi J. 2010. Caractérisation hydrodynamique et hydrogéochimique des aquifères fissurés de la région de Toumodi (centre de la Côte d'ivoire). Journal of Environmental Hydrology, 18: Paper 26, 15p. https://docplayer.fr/77386681Caracterisation-hydrodynamique-ethydrogeochimique-des-aquiferesfissures-de-la-region-de-toumodi-centrede-la-cote-d-ivoire.html
Yoboué KE, Yao-Kouamé A, Alui KA. 2010. Evolution Pédogéochimique et Minéralogique au Cours de l'altération des Formations du Complexe Volcanosédimentaire d'Anikro et de Kahankro (Toumodi) dans le Centre-Sud de la Côte d'Ivoire. European Journal of Scientific Research, 40(1): $60-72$.

Yoboué KE, Bongoua-Devisme AJ, Kouadio KP, Yao-kouamé A. 2014. Minéralogie de la fraction argileuse des sols brunifiés de Kahankro et Anikro (Toumodi) dans le Centre Sud de la Côte d'Ivoire. Int. J. Biol. Chem. Sci., 8(3) : 1269-1280. DOI: http://dx.doi.org/10.4314/ijbcs.v8i3.40

Yoboué KE, Kouadio KP, Blé LO, YaoKouamé A. 2018. Caractéristiques Morphopédologiques et Géochimiques des Sols Brunifiés de Anikro et de Kahankro (Centre-Sud de la Côte d'Ivoire). European Scientific Journal, 14(3): 281-300. DOI: http://dx.doi.org/10.19044/esj.2018.v14n 3. 